\title{
A MymA bioactivated thioalkyl-benzoxazole prodrug family active against Mycobacterium tuberculosis
}

\author{
Abraham L. Moure, ${ }^{1,2^{*}}$ Gagandeep Narula, ${ }^{2,3}$ Flavia Sorrentino, ${ }^{1,2}$ Adama Bojang, ${ }^{2,3}$ \\ Clement K.M. Tsui, ${ }^{2}$ Carine Sao Emani, ${ }^{2}$ Esther Porras-De Francisco, ${ }^{1}$ Beatriz Díaz, ${ }^{1}$ María \\ José Rebollo-López, ${ }^{1}$ Pedro Alfonso Torres-Gómez, ${ }^{1}$ Eva María López-Román, ${ }^{1}$ Isabel \\ Camino, ${ }^{1}$ Patricia Casado Castro, ${ }^{1}$ Laura Guijarro López, ${ }^{1}$ Fátima Ortega, ${ }^{1}$ Lluis Ballell, ${ }^{1}$ \\ David Barros-Aguirre, ${ }^{1}$ Modesto Remuiñán Blanco, ${ }^{1 *}$ Yossef Av-Gay ${ }^{2,3^{*}}$ \\ Diseases of the Developing World (DDW), Global Health Catalyst, GlaxoSmithKline, \\ Severo Ochoa 2, 28760 Tres Cantos Madrid, Spain, ${ }^{1}$ Division of Infectious Diseases, \\ Departments of Medicine ${ }^{2}$ and Microbiology and Immunology ${ }^{3}$, Life Sciences Institute, \\ University of British Columbia, Vancouver, British Columbia, Canada \\ Correspondence: yossi@mail.ubc.ca, modesto.b.remuinan@gsk.com, \\ abraham.x.lopez@gsk.com
}




\section{Table of contents :}

General antimicrobial activity assay for 1

Safety report of 1

Site of metabolism for compound for 1

. .56

GSH trapping experiment for 1

.57

CYP3A4 inhibition assay with compound 1

.57

Chemical structures of sulfoxide $1 \mathrm{a}$ and sulfone $1 \mathrm{~b}$ .S7

Additional experimental data for analogs 2-49 .58

HPLC determination for compounds 1 and 46 S10 


\section{General antimicrobial activity assay}

Whole-cell antimicrobial activity was determined by broth microdilution using the Clinical and Laboratory Standards Institute (CLSI) recommended procedure, Document M7-A7, "Methods for Dilution Susceptibility Tests for Bacteria that Grow Aerobically". Selected compounds were evaluated against a panel of Gram-positive and Gram-negative organisms. Minimum inhibitory concentration (MIC) values were determined as the lowest concentration of compound producing $90 \%$ decrease in fluorescence observed.

Table S1. Activity of 1 against a panel of gram positive and gram negative bacteria.

\begin{tabular}{rlcc}
\cline { 3 - 3 } & & \multicolumn{2}{c}{ Compound MIC $(\mu \mathrm{g} / \mathrm{mL})$} \\
\cline { 2 - 3 } & Isolate & \multicolumn{1}{c}{ Control } \\
\hline 1 & Staph.aureus WCUH29 & $>128$ & Ceftazidime* \\
2 & Staph.aureus PVL-6 & $>128$ & $>16$ \\
3 & Strep.pneumoniae ERY2 & $>128$ & 0.125 \\
4 & Strep.pneumoniae TPS3 & 128 & 4 \\
5 & Strep.pyogenes 1308007P & $>128$ & 0.125 \\
6 & Haemophilus influenzae H128 & 64 & $\leq 0.016$ \\
7 & E.coli 7623 & $>128$ & 0.25 \\
8 & E.coli 7623 TolC- (efflux mutant) & 128 & 0.125 \\
9 & E.coli D21 Parent & $>128$ & 2 \\
10 & E.coli D22 lpxC- (OM deficient) & $>128$ & 0.03 \\
11 & E.coli NCTC 13441 (in vivo) & $>128$ & $>16$ \\
12 & K1.pneumoniae 1161486 & $>128$ & 0.125 \\
13 & Kl.pneumoniae VA-361 (in vivo) & $>128$ & $>16$ \\
14 & Pse.aeruginosa PAO1 (MV) & $>128$ & 2 \\
15 & Pse.aeruginosa SR27001 (in vivo) & $>128$ & $>16$ \\
16 & Acin.baumannii BM4454 & $>128$ & 8 \\
17 & Acin.baumannii 1485247 (in vivo) & $>128$ & $>16$ \\
\hline 18 & Moraxella catharrhalis 25430 & $>15.5$ & $0.81^{* *}$ \\
\hline 19 & M. smegmatis MC 155 & $>15.5$ & $1.62^{* *}$ \\
\hline 20 & M. Bovis BCG*** & $>15.5$ & $1.62^{* *}$ \\
\hline 21 & M. avium avium clinical isolate*** & $>15.5$ & $1.62^{* *}$ \\
\hline 22 & MAC (M. avium complex) clinical isolate*** & $>15.5$ & $1.62^{* *}$ \\
\hline 23 & M. intracellularae clinical isolate*** & $>15.5$ & $1.62^{* *}$ \\
\hline & $*$ Ceftazidime within historical quality control range & \\
& $* *$ Gentamicin control & & \\
& $* * *$ Resazurin assay & & \\
& & & \\
\hline
\end{tabular}




\section{Safety report}

$\mathbf{1}$ was profiled looking for specific safety alerts, focus on cytotoxicity, cardiovascular issues and human secondary pharamacological pannel.

The cytotoxic effect ${ }^{1}$ of the compounds is measured by a three parameter automated imaging cell based assay which is performed in human liver derived HepG2 cells using fluorescent staining. The key parameters measured in this assay are nuclear size, mitochondrial membrane potential and plasma membrane permeability.

In addition, toxicity in the cardiovascular system is principally measured by hERG channel. ${ }^{2}$ This channel is a voltage-gated potassium channel strongly expressed in heart and involved in repolarization of the cardiac action potential so the screening for inhibition of $h E R G$ is essential to go ahead with a compound.

A human secondary pharmacological pannel was run, consists of in vitro assays designed to measure the effect of test compounds on receptor sites, ion channel, transporters and anzymes, and in phenotypic assays. ${ }^{3}$

\footnotetext{
${ }^{1}$ Sison-Young RL, Lauschke VM, Johann E, Alexandre E, Antherieu S, Aerts H, Gerets HHJ, Labbe G, Hoët D, Dorau M, Schofield CA, Lovatt CA, Holder JC, Stahl SH, Richert L, Kitteringham NR, Jones RP, Elmasry M, Weaver RJ, Hewitt PG, Ingelman-Sundberg M, Goldring CE, Park BK. A multicenter assessment of single-cell models aligned to standard measures of cell health for prediction of acute hepatotoxicity. Arch Toxicol. 2017 Mar;91(3):1385-1400.

2 2. Priest BT, Bell IM, Garcia ML. Role of hERG potassium channel assays in drug development. Channels (Austin). 2008 Mar-Apr;2(2):87-93

3 3. Papoian T, Chiu HJ, Elayan I, Jagadeesh G, Khan I, Laniyonu AA, Li CX, Saulnier M, Simpson N, Yang B. Secondary pharmacology data to assess potential off-target activity of new drugs: A regulatory perspective. Nat Rev Drug Discov. 2015 Apr;14(4):294.
} 
Table S2. Principal safety alerts of $\mathbf{1}$.

\begin{tabular}{|c|c|c|c|c|}
\hline & Target & $\begin{array}{c}\text { Type of } \\
\text { assay }\end{array}$ & Mode & $I_{50}(\mu \mathrm{M})$ \\
\hline \multirow[t]{3}{*}{ Cytotoxicity } & Cell Health & Pheno & $\begin{array}{l}\text { Mitochondrial } \\
\text { Integrity }\end{array}$ & 31.6 \\
\hline & Cell Health & Pheno & $\begin{array}{c}\text { Membrane } \\
\text { Permeability }\end{array}$ & 158.5 \\
\hline & Cell Health & Pheno & Nucleus (size) & 125.9 \\
\hline \multirow[t]{7}{*}{ Cardiovascular } & PDE4B & ENZ & Ant & 100.0 \\
\hline & KCNQ1/minK & IC & Block & 25.1 \\
\hline & Kv1.5 & IC & Block & 50.1 \\
\hline & Vasopressin 1a & 7-TM & Ant & 50.1 \\
\hline & hERG & IC & Block & \\
\hline & Cav1.2 & IC & Block & 100 \\
\hline & Nav1.5 & IC & Block & 100 \\
\hline \multirow[t]{24}{*}{ SNC } & GABA-A $\alpha 1$ & IC & PMod & 50.1 \\
\hline & GABA-A $\alpha 1$ & IC & $\mathrm{Ag}$ & 100.0 \\
\hline & GABA-A $\alpha 1$ & IC & Ant & 100.0 \\
\hline & Dopamine 1 & 7-TM & Ant & 100.0 \\
\hline & Histamine 1 & 7-TM & Ant & 25.1 \\
\hline & Muscarine 1 & 7-TM & $\mathrm{Ag}$ & 50.1 \\
\hline & Muscarine 1 & 7-TM & Ant & 50.1 \\
\hline & Neurokinin 1 & 7-TM & Ant & 25.1 \\
\hline & Serotonin 1B & 7-TM & $\mathrm{Ag}$ & 100.0 \\
\hline & Serotonin 2A & 7-TM & $\mathrm{Ag}$ & 100.0 \\
\hline & Serotonin $2 A$ & 7-TM & Ant & 100.0 \\
\hline & Serotonin $2 \mathrm{C}$ & 7-TM & $\mathrm{Ag}$ & 100.0 \\
\hline & Serotonin $2 \mathrm{C}$ & 7-TM & Ant & 100.0 \\
\hline & Serotonin 2B & 7-TM & $\mathrm{Ag}$ & 100.0 \\
\hline & Serotonin 3 & IC & Open & 50.1 \\
\hline & Serotonin 3 & IC & Block & 25.1 \\
\hline & Serotonin - SERT & TRAN & Ant & 100.0 \\
\hline & Adenosine $2 \mathrm{a}$ & 7-TM & $\mathrm{Ag}$ & 100.0 \\
\hline & Adrenergic $\alpha 1 b$ & 7-TM & Ant & 25.1 \\
\hline & $\operatorname{cox} 2$ & ENZ & Block & 100.0 \\
\hline & Noradrenaline - NET & TRAN & Ant & 100.0 \\
\hline & PDE3A & ENZ & Inh & 100.0 \\
\hline & AChEase & ENZ & Inh & 100.0 \\
\hline & $\begin{array}{c}\text { Monoamine oxidase } \\
\text { A }\end{array}$ & ENZ & Inh & 100.0 \\
\hline PLD & Phospholipidosis & Pheno & Accum & 100.0 \\
\hline \multirow{6}{*}{ Hepatotoxicity- DDI } & CYP3A4 & ENZ & Ant & 39.8 \\
\hline & $\begin{array}{l}\text { CYP3A4 Mechanism- } \\
\text { dependent Inhibition }\end{array}$ & ENZ & $\begin{array}{l}\text { plC50 fold- } \\
\text { shift }\end{array}$ & N/A \\
\hline & OATP1B1 & TRAN & Inh & 50.1 \\
\hline & AhR & NR & $\mathrm{Ag}$ & 100.0 \\
\hline & BSEP & TRAN & Inh & 39.8 \\
\hline & PXR & NR & $\mathrm{Ag}$ & 12.6 \\
\hline Immune respond & $\operatorname{MrgX2}$ & 7-TM & $\mathrm{Ag}$ & 100.0 \\
\hline Calcium movilization & $\begin{array}{l}\text { CHO null host } \\
\text { Calcium }\end{array}$ & Pheno & $\mathrm{Ag}$ & 100.0 \\
\hline
\end{tabular}


Figure S1. Site of metabolism for compound 1. MetaSite 6.0; Molecular Discovery Ltd

$\checkmark$ The position in blue circle is the most reactive position.

$\checkmark$ Then the most likely sites of metabolism are classified from dark red (most likely) to light red (less likely), with the percentage of likelihood as a number on the position.

$\checkmark$ The "Liver" consensus SoM model has been used (includes CYP2C9, CYP2D6, CYP3A4).

$\checkmark$ Compound 1 was not recognized as substrate for AOX and FMO3.

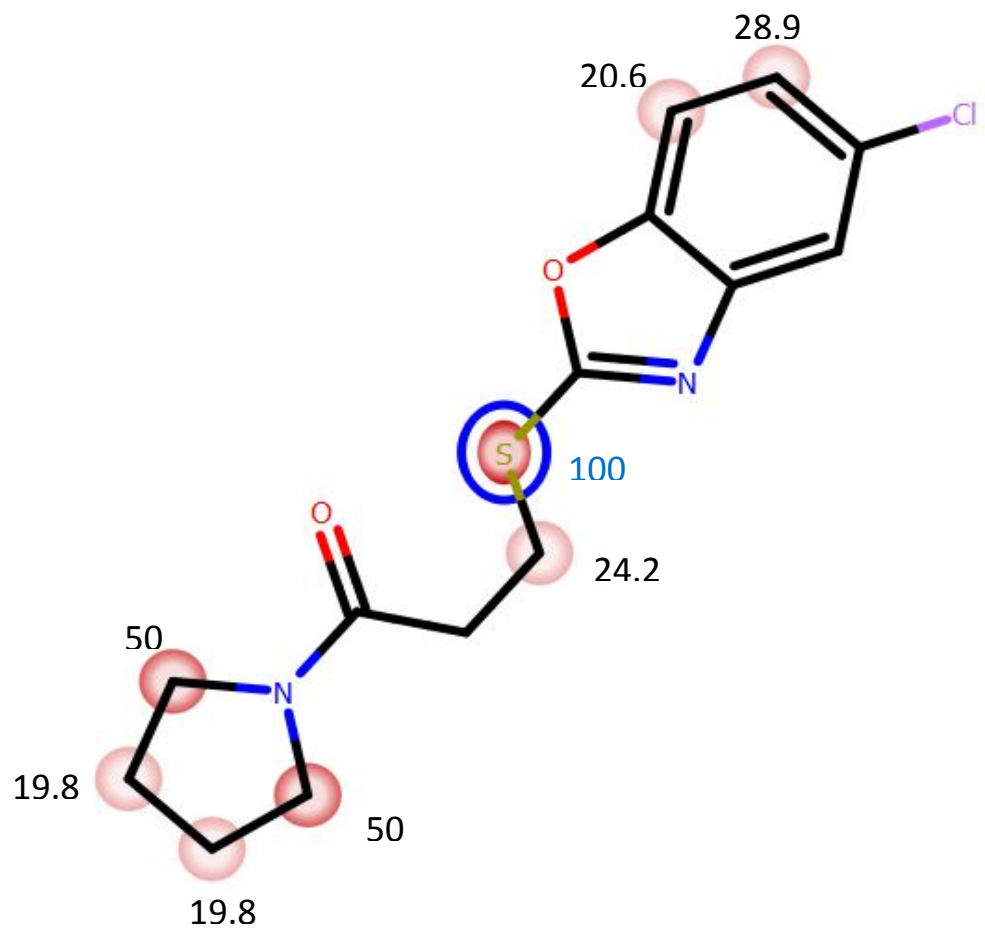

METASITE_METID_MECHS

S-Dealkylation

Thioetheric S-Oxidation

Aliphatic Hydroxylation

$\mathrm{N}$-Dealkylation

Iminium Formation (5-member

ring)

Dehydrogenation

Aliphatic Carbonylation

Aromatic Hydroxylation

Dehydrogenation

Aromatic Hydroxylation

Aliphatic Hydroxylation
METASITE_METID_SCORE

100

100

50

50

50

50

50

28.8889

24.2064

20.5556

19.7619 
Table S3. GSH trapping experiment

\begin{tabular}{ccccc}
\hline Test Article & Scan & $\begin{array}{c}\text { Potential Reactive } \\
\text { Metabolites Identified }\end{array}$ & $\mathbf{m} / \mathbf{z}$ & Comment \\
\hline \multirow{2}{*}{ Ticlopidine } & Precursor & Yes & 585 & Positive control \\
& Neutral loss & Yes & 587 & Positive control \\
\hline \multirow{2}{*}{ Compound 1 } & Precursor & No & & \\
& Neutral loss & No & & \\
\hline
\end{tabular}

Table S4. CYP3A4 inhibition assay with compound 1

\begin{tabular}{|c|c|c|c|}
\hline Compound & $\mathrm{SPI}^{\mathrm{a}}\left(\mathrm{pIC}_{50}\right)$ & $\mathrm{CPI}^{\mathrm{b}}\left(\mathrm{pIC}_{50}\right)$ & MDI fold shift \\
\hline $\mathbf{1}$ & $<4.40$ & $<4.40$ & 1 \\
\hline
\end{tabular}

${ }^{\mathrm{a} S u b s t r a t e}$ pre-incubation. ${ }^{\mathrm{b}}$ Cofactor pre-íncubation

Figure S2. Chemical structures of sulfoxide 1a and sulfone 1b<smiles>O=C(CCS(=O)c1nc2cc(Cl)ccc2o1)N1CCCC1</smiles>

1a

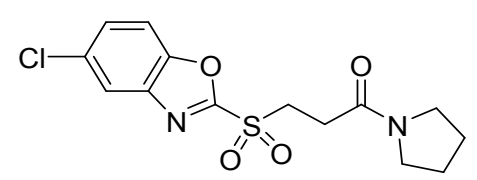

$1 b$ 
Table S5. Additional experimental data for analogues

\begin{tabular}{|c|c|c|c|}
\hline $\begin{array}{l}\text { Compoun } \\
\text { d number }\end{array}$ & $\begin{array}{c}\text { In-vitro MIC } \\
(\mu \mathrm{M})\end{array}$ & $\begin{array}{l}\text { HepG2 } \\
\mathrm{IC}_{50}(\mu \mathrm{M})\end{array}$ & $\begin{array}{c}\text { Solubility } \\
\qquad(\mu \mathrm{M})\end{array}$ \\
\hline 2 & $>80$ & $>100$ & $\geq 532$ \\
\hline 3 & $>80$ & $>100$ & 253 \\
\hline 4 & $>80$ & $>100$ & $\geq 418$ \\
\hline 5 & $>80$ & $>100$ & $\geq 393$ \\
\hline 6 & 10 & $>100$ & $\geq 673$ \\
\hline 7 & $>80$ & $>100$ & $\geq 400$ \\
\hline 8 & 10 & $>100$ & 328 \\
\hline 9 & $>80$ & $>100$ & n.d. \\
\hline 10 & $>80$ & $>100$ & $\geq 458$ \\
\hline 11 & 5 & $>100$ & 199 \\
\hline 12 & 10 & $>100$ & $\geq 591$ \\
\hline 13 & 5 & $>100$ & $\geq 445$ \\
\hline 14 & 10 & $>100$ & $\geq 816$ \\
\hline 15 & 20 & $>100$ & n.d. \\
\hline 16 & 5 & $>100$ & $>439$ \\
\hline 17 & $>80$ & $>100$ & $>440$ \\
\hline 18 & 2.5 & $>100$ & $>383$ \\
\hline 19 & $>80$ & $>100$ & 66 \\
\hline 20 & $>80$ & $>100$ & $>365$ \\
\hline 21 & $>80$ & $>100$ & $>386$ \\
\hline 22 & $>80$ & $>100$ & $>403$ \\
\hline 23 & $>80$ & $>100$ & $>410$ \\
\hline 24 & 80 & $>100$ & $>420$ \\
\hline 25 & $>80$ & $>100$ & $>597$ \\
\hline 26 & $>80$ & $>100$ & $>450$ \\
\hline 27 & $>80$ & $>100$ & 351 \\
\hline 28 & 10 & $>100$ & $>422$ \\
\hline 29 & 5 & $>100$ & 344 \\
\hline 30 & 40 & $>100$ & $>510$ \\
\hline 31 & $>80$ & $>100$ & 248 \\
\hline 32 & $>80$ & 79.4 & $>437$ \\
\hline
\end{tabular}


Table S5. Additional experimental data for analogues (cont.)

\begin{tabular}{cccc}
\hline $\begin{array}{c}\text { Compoun } \\
\text { d number }\end{array}$ & $\begin{array}{c}\text { In-vitro MIC } \\
(\mu \mathrm{M})\end{array}$ & $\begin{array}{c}\text { HepG2 } \\
\mathrm{IC}_{50}(\mu \mathrm{M})\end{array}$ & $\begin{array}{c}\text { Solubility } \\
(\mu \mathrm{M})\end{array}$ \\
\hline 33 & $>80$ & $>100$ & $>456$ \\
34 & n.d. & $>100$ & 49 \\
35 & $>80$ & $>100$ & 337 \\
36 & n.d. & $>100$ & 359 \\
37 & n.d. & $>100$ & 170 \\
38 & $>80$ & $>100$ & 71 \\
39 & n.d. & $>100$ & 303 \\
40 & $>80$ & $>100$ & $>597$ \\
41 & $>80$ & $>100$ & $>495$ \\
42 & 80 & $>100$ & $>295$ \\
43 & $>80$ & $>100$ & $>435$ \\
44 & $>80$ & $>100$ & 31 \\
45 & $>80$ & $>100$ & 369 \\
46 & 40 & $>100$ & $>328$ \\
47 & 80 & $>100$ & 343 \\
48 & $>80$ & $>100$ & 332 \\
49 & $>80$ & $>100$ & $>396$ \\
& & &
\end{tabular}


Compound 1

Sample amount: $1 \mathrm{mg}$

Solvent: DMSO

Concentration: $1 \mathrm{mg} / \mathrm{mL}$

Column: Acquity UPLC BEH C18 1.7u 3x50mm

Method: $0.1 \%$ Formic Acid in Water $/ 0.1 \%$ Formic Acid in ACN

Initial $\quad 95: 5$

$0.0-1.4 \min 0: 100$

$1.4-1.9 \min 0: 100$

$1.9-2.0 \mathrm{~min} 95: 5$

Flow: $0.8 \mathrm{~mL} / \mathrm{min}$

Inj. Vol.: $0.2 \mathrm{uL}$

$T^{\mathrm{a}}: 50^{\circ} \mathrm{C}$

\begin{tabular}{|c|c|c|c|c|c|}
\hline $\begin{array}{c}\text { Retention } \\
\text { Time(min) }\end{array}$ & Molecular Formula & $\begin{array}{c}\text { Monoisotopic } \\
\text { Mass }\end{array}$ & {$[\mathrm{M}+\mathrm{H}]^{+}$} & {$[\mathrm{M}-\mathrm{H}]$} & $\begin{array}{c}\text { Purity } \\
(\mathbf{2 5 2 n m})\end{array}$ \\
\hline 1.29 & C14H15CIN2O2S & 310.05 & 311 & & $>95$ \\
\hline
\end{tabular}

Chromatograms \& Spectra:
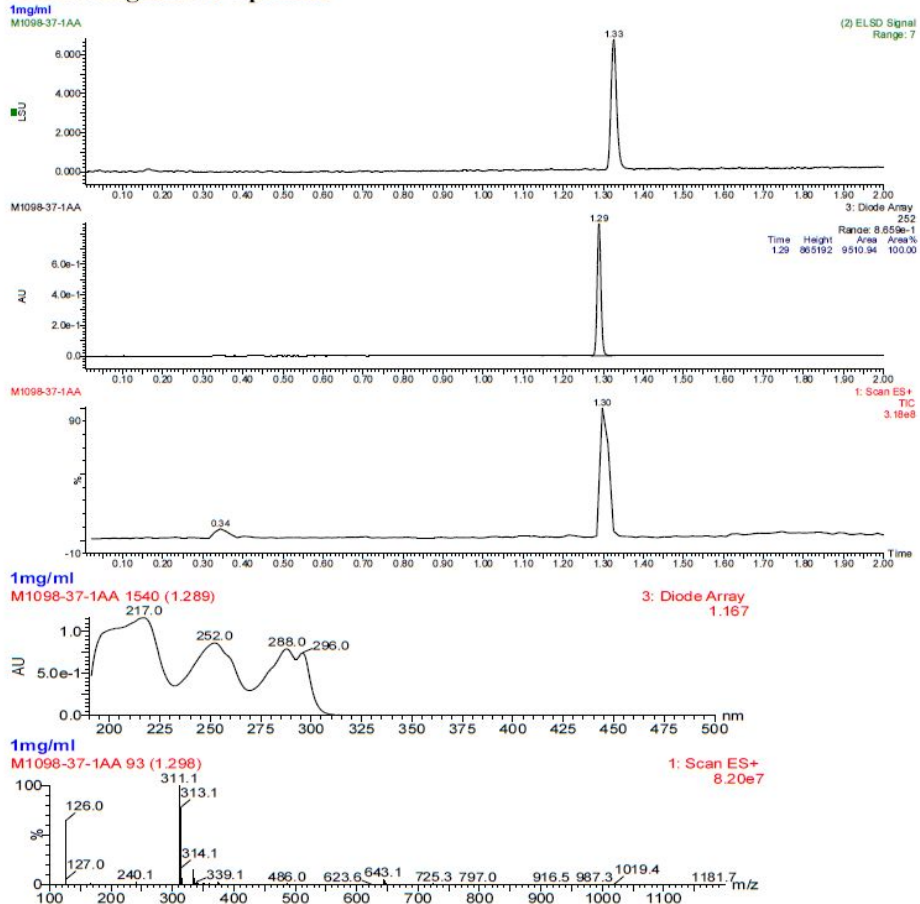


\section{Compound 46}

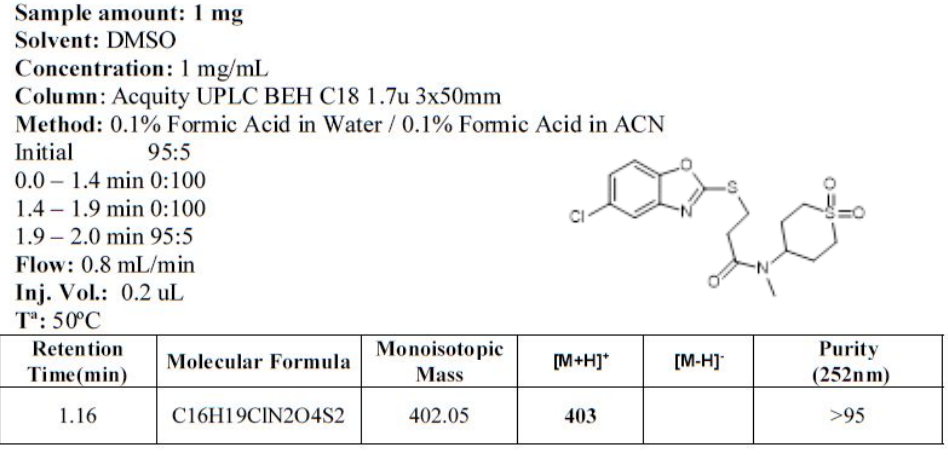

Chromatograms \& Spectra:

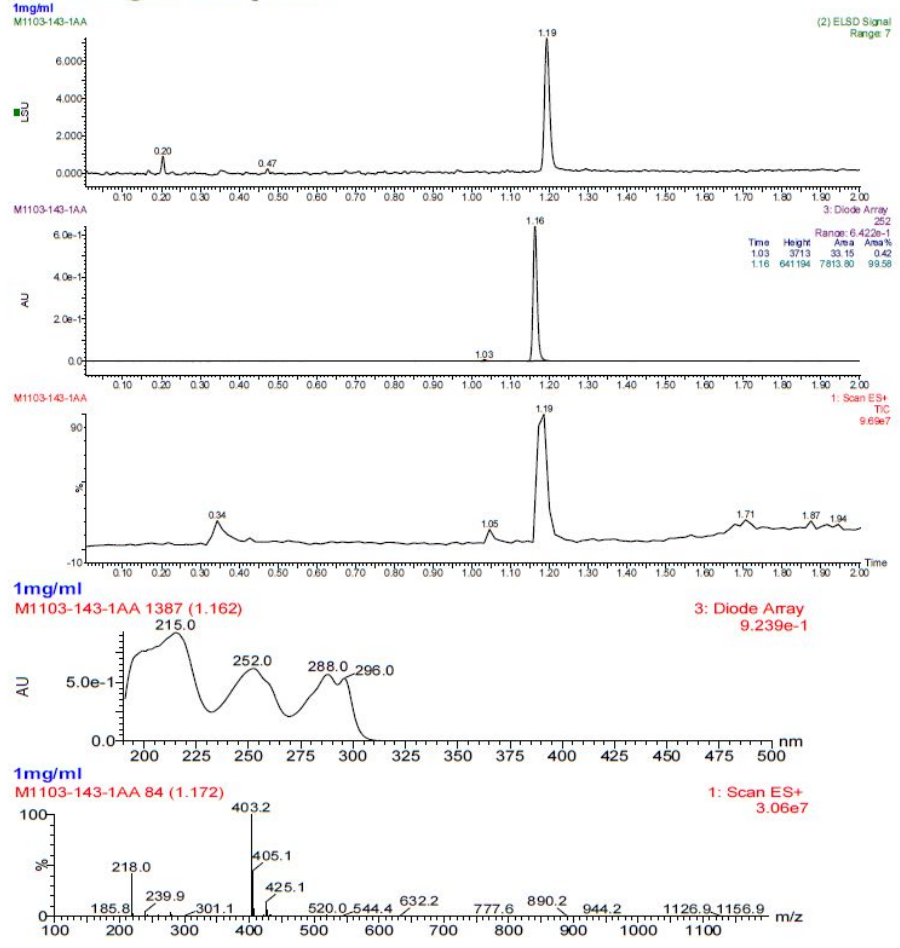

